\title{
Torque Control of Electric Power Steering Systems Based on Improved Active Disturbance Rejection Control
}

\author{
Shaodan Na, ${ }^{1}$ Zhipeng Li $\mathbb{D}^{1},{ }^{1}$ Feng Qiu, ${ }^{2}$ and Chao Zhang ${ }^{1}$ \\ ${ }^{1}$ School of Traffic and Transportation, Northeast Forestry University, Harbin 150040, China \\ ${ }^{2}$ College of Mechanical and Electrical Engineering, Northeast Forestry University, Harbin 150040, China \\ Correspondence should be addressed to Zhipeng Li; lzp_100@126.com
}

Received 15 January 2020; Accepted 25 March 2020; Published 29 April 2020

Academic Editor: Salvatore Strano

Copyright (c) 2020 Shaodan Na et al. This is an open access article distributed under the Creative Commons Attribution License, which permits unrestricted use, distribution, and reproduction in any medium, provided the original work is properly cited.

In the electric power steering (EPS) system, low-frequency disturbances such as road resistance, irregular mechanical friction, and changing motor parameters can cause steering wheel torque fluctuation and discontinuity. In order to improve the steering wheel torque smoothness, an improved torque control method of an EPS motor is proposed in the paper. A target torque algorithm is established, which is related to steering process parameters such as steering wheel angle and angular speed. Then, a target torque closed-loop control strategy based on the improved ADRC is designed to estimate and compensate the internal and external disturbance of the system, so as to reduce the impact of the disturbance on the steering torque. The simulation results show that the responsiveness and anti-interference ability of the improved ADRC is better than that of the conventional ADRC and PI. The vehicle experiment shows that the proposed control method has good motor current stability, steering torque smoothness, and flexibility when there is low-frequency disturbance.

\section{Introduction}

An electric power steering (EPS) system has the advantages of safety, energy saving, and comfortable steering [1], which has gradually replaced mechanical and hydraulic power systems to achieve assist power steering function in the steering system [2-4]. However, the EPS system also causes some issues. For example, the application of motor and deceleration mechanism in EPS inevitably increases the inertia of the steering system and introduces unknown additional friction. The EPS controller sampling noise and imprecision of the control model will also cause disturbance. These issues can cause discontinuity and fluctuation for steering torque, especially when the steering resistance torque changes, greatly caused by unknown road bumps. The frequency of the above disturbance is very low, even close to the range of input signal. It is difficult to filter out by means of a filter. Therefore, it is important to improve the responsiveness and keep stability when unknown disturbance occurs.
In recent years, many control strategies are proposed to improve the steering feel and torque responsiveness for EPS control [5-7]. In [8], the PID strategy is used to establish a torque closed loop, in which the differential operation produces high-frequency noise that drowns out the differential signals. Therefore, designers tend to use the PI strategy in practical applications. However, when the unknown load disturbance varies dramatically, the PI strategy quickly increases the power gain to shorten the response time, which easily arouses the system fluctuation, resulting in uneven steering torque.

With the development of modern control theory technology, many intelligent control methods have been applied in EPS torque control. In [9-11], the genetic algorithm and particle swarm optimization are used to find the optimal gain parameters. In [12], the ant colony optimization and particle swarm optimization are proposed to find the optimal PID parameters, which can improve the responsiveness and stability. However, these algorithms all belong to the random algorithm, which has some problems such as 
local optimal solution, premature convergence, and large computation.

Some scholars adopt the adaptive sliding mode observer to track the torque variation to ensure robustness in the steering system, and the high-frequency noise of differential signal is eliminated by using a Kalman filter [13-15]. The sliding mode control is a bang-bang control, which will cause torque fluctuation and affect the smoothness. In addition, the calculation of the Kalman filter is relatively large, so it is difficult to be applied in practice.

Many other scholars have applied the $\mathrm{H}-\infty$ theory to torque control. In [16-18], the $\mathrm{H}-\infty$ controller of the steering system model is established. In [19], H-2 and H-O are combined to design the observer to find the optimal solution. The key of $\mathrm{H}-\infty$ is to find out the frequency range of different errors in the system model and to determine the index of the optimal solution. However, the index values are always based on design experiences, and the control accuracy is affected by the system model which varies by vehicle type. Therefore, the algorithm is not desirable in engineering application.

The ctive disturbance rejection control (ADRC) strategy was proposed in the late 1990s [20], which not only absorbed the modern control theory of "system internal mechanism description" but is also based on the control strategy of "eliminating error by error." The core of ADRC is used to unify the internal and external disturbance of the system into a total disturbance, which is estimated by an extended state observer (ESO) and compensated by closed-loop control controller [21]. Then, the system can have good responsiveness and anti-interference ability in the unknown disturbance environment.

Due to the nonlinearity and unpredictability of disturbance, the nonlinear extended state observer (NESO) is used to ensure the fast response. However, the calculation of NESO is very large, which is not suitable for engineering application. The calculation of the linear extended state observer (LESO) is small, but the responsiveness of LESO is poor. The control system needs to consider both responsiveness and calculation.

The torque control accuracy of the DC permanent magnet motor is studied under low-frequency disturbance in this paper. The motor structure is simple, which contains a small amount of the unknown parameters, resulting in high accuracy for disturbance estimation. In addition, with the estimated frequency of disturbance becoming low, the estimated burden of ESO becomes small. Therefore, the proposed ADRC strategy has good control effect on lowfrequency disturbance.

This paper proposes a torque control method based on improved ADRC. The system disturbance is estimated and compensated by a parallel linear extended state observer (PLESO) instead of the conventional ESO to ensure the good responsiveness and avoid excessive calculation.

The rest of the paper is organized as follows. In Section 2, the mathematical models of the steering system and target steering torque algorithm are given. In Section 3, the tracking differentiators (TD), P-LESO, and feedback closedloop control equation are applied to the steering torque control, and simulation verification is performed. In Section 4 , the vehicle experiment verifies the effectiveness of the algorithm is presented. Finally, Section 5 provides some good conclusions.

\section{Dynamics Analysis of the EPS System}

2.1. Simplified Model of the EPS System. Figure 1 shows a simplified model of the EPS system, which includes two ends of the torque bar connected with the steering wheel and the steering column, respectively. The torque sensor installed on the torque bar detects a shape variable to provide the signal for the ECU controller, which can control the motor to supply assist torque. The motor assist torque is amplified by the deceleration mechanism to overcome the internal friction and road resistance torque [22].

Torque equation for the upper end of the torque bar can be expressed as

$$
T_{\mathrm{sw}}-T_{s}=c_{\mathrm{sw}} \omega+J_{\mathrm{sw}} a_{\mathrm{sw}},
$$

where $T_{\mathrm{sw}}$ is the steering wheel torque; $T_{s}$ is the torque of the torque bar, which is measured by the torque sensor; $c_{\mathrm{sw}}$ is the steering wheel damping; $\omega$ is the angular speed of the steering wheel; $J_{\mathrm{sw}}$ is the rotary inertia of the steering wheel; and $a_{\mathrm{sw}}$ is the steering wheel angular acceleration.

In fact, the moment of inertia $J_{s w}$ and the steering wheel damping $c_{\mathrm{sw}}$ are too small to be considered. Therefore, equation (1) can be simplified as

$$
T_{s} \approx T_{\mathrm{sw}}
$$

Torque equation for the lower end of the torque bar can be expressed as

$$
G T_{e}+T_{s}=J_{p} a_{p}+c_{p} \omega_{p}+T_{r},
$$

where $G$ is the reduction ratio of the deceleration mechanism; $T_{e}$ is the motor electromagnetic torque; $J_{p}$ is the rotary inertia of the steering column; $a_{p}$ is the angular acceleration of the steering column; $c_{p}$ is the steering column damping; $\omega_{p}$ is the angular speed of the steering column; and $T_{r}$ is the road resistance torque. In addition, the motor electromagnetic torque can be expressed as follows:

$$
T_{e}=K_{m} \cdot i
$$

where $K_{m}$ is the motor torque constant and $i$ is the motor current.

As the motor is rigidly connected with the deceleration mechanism, the $\omega_{m}$ is expressed as the angular speed of the motor and then $\omega_{p}$ can be written as

$$
\omega_{m}=G \omega_{p} .
$$

Therefore, by means of equations (2) to (5), the equation of steering wheel torque can be expressed as

$$
T_{\mathrm{sw}}=-G K_{m} i+T_{r}+J_{p} a_{p}+c_{p} \frac{\omega_{m}}{G} .
$$

It can be seen that the steering wheel torque is greatly affected by the road disturbance and system damping. The external disturbance can be expressed as a function $a(t)$. 


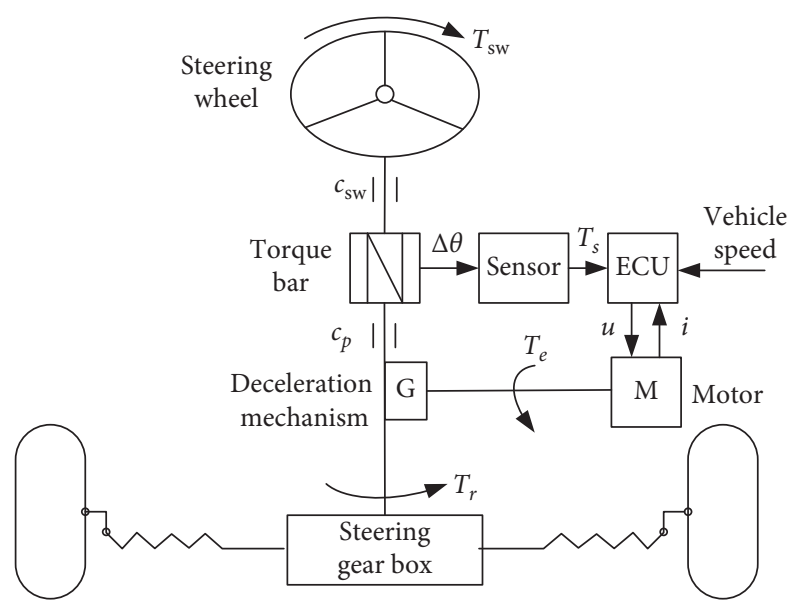

Figure 1: Simplified model of EPS system.

To ensure smooth and stable steering torque, the influence of other interference terms needs to be compensated by controlling the motor current.

The simplified mathematical model of the motor can be expressed as

$$
u=R i+p L_{m} i+K_{e} \omega_{m}
$$

where $u$ is the motor voltage; $R$ is the equivalent resistance of the motor armature; $p$ is the differential operator; $L_{m}$ is the motor inductance; and $K_{e}$ is the back-EMF coefficient of the motor.

The main parameters in the above equation are shown in Table 1.

2.2. Target Steering Torque Algorithm. In order to avoid the influence of nonmajor factors in the algorithm, the target torque control algorithm used in this study is established under the following conditions: the vehicle steering system structure is rigidly connected without force deformation in the steering mechanism of the vehicle; the tire side-slip angle during steering is ignored.

Generally, the requirement of the vehicle to the steering wheel torque varies with the driving condition. When the vehicle is driving at low speed or stationary, the steering torque should be flexible and light. As the vehicle speed increases, the steering torque should be appropriately increased to ensure that the vehicle can resist the interference of lateral forces when driving in a straight line. In the case of constant vehicle speed, as the steering wheel angle increases, the steering torque should increase to ensure the steering feel; when the angular speed of the steering wheel is large, it means that the direction of the vehicle is changing rapidly, and the steering torque should be appropriately increased to ensure the stability of the vehicle operation. Therefore, the target steering torque control algorithm can be expressed as

$$
\begin{cases}T_{\mathrm{sw}}^{*}=K_{\theta} \cdot\left(K_{v} \cdot v+1\right) \cdot\left(\theta-\theta_{d}\right), & \omega<\omega_{k}, \\ T_{\mathrm{sw}}^{*}=K_{\theta} \cdot\left(K_{v} \cdot v+1\right) \cdot\left[\left(\theta-\theta_{d}\right)+K_{\omega} \cdot\left(\omega-\omega_{k}\right)\right], & \omega \geq \omega_{k},\end{cases}
$$

TABLE 1: Main parameters of the steering system.

\begin{tabular}{lcc}
\hline Parameters & Unit & Value \\
\hline$G$ & - & 16.5 \\
$J_{p}$ & $\mathrm{~kg} \cdot \mathrm{m}^{2}$ & 0.041 \\
$c_{p}$ & $\mathrm{~N} \cdot \mathrm{m} \cdot \mathrm{s} \cdot \mathrm{rad}^{-1}$ & $0.25-0.4$ \\
$R$ & $\Omega$ & $0.16-0.3$ \\
$L_{m}$ & $\mathrm{mH}$ & 0.1 \\
$K_{e}$ & $\mathrm{~V} \cdot \mathrm{s} \cdot \mathrm{rad}$ & 0.053 \\
$K_{m}$ & $\mathrm{~N} \cdot \mathrm{m} \cdot \mathrm{A}^{-1}$ & 0.053 \\
\hline
\end{tabular}

where $T_{\text {sw }}^{*}$ is the target steering torque; $v$ is the vehicle speed; $\theta$ is the angle of the steering wheel; $\theta_{d}$ is the steering wheel angle dead zone; $\omega$ is the angular speed of the steering wheel; $\omega_{k}$ is the limiting angular speed of the steering wheel; and $K_{v}$, $K_{\theta}$, and $K_{\omega}$ are the control parameters that directly affect the steering feel of the driver. The corresponding target steering torque control algorithm can be adjusted according to different vehicle types, driving preferences, and actual working conditions.

\section{Design of Improved ADRC Torque Control Strategy}

Figure 2 shows the block diagrams of EPS motor control based on ADRC, which includes an outer torque closed loop and an inner current closed loop. The angle torque sensor measures the angle and torque of the steering wheel in real time. The tracking differentiator (TD) calculates the angle to obtain the angular speed and the transition process of angle, which are used by the target torque algorithm to get the target torque of the steering wheel. The torque of the steering wheel is affected by factors such as road resistance, mechanical friction, and motor assist torque. According to the difference between the target torque and the measured torque of the steering wheel, the motor current is controlled by ADRC to provide the appropriate assist torque to achieve torque closed-loop control. Meanwhile, the unknown road resistance, irregular mechanical friction, and changing motor parameters cause interference to the current control. Therefore, the ADRC uses LESO to estimate these disturbances and compensates it in the current closed-loop control.

3.1. Angular Speed Calculation with TD. In the target torque algorithm, both the vehicle speed and steering wheel angles are measured by the corresponding sensor. The angular speed is usually obtained by differentiating the angle signal, which has a lot of differential noise. The transfer function of conventional angular speed calculation is

$$
\begin{aligned}
\omega_{c}(s) & =\frac{s}{T s+1} \theta(s) \\
& =\frac{1}{T}\left(\theta(s)-\frac{\theta(s)}{T s+1}\right),
\end{aligned}
$$

where $\omega_{c}$ is the angular speed obtained by conventional differential calculations; $\theta$ is the steering wheel angle; and $T$ is a time constant representing the system step size. 


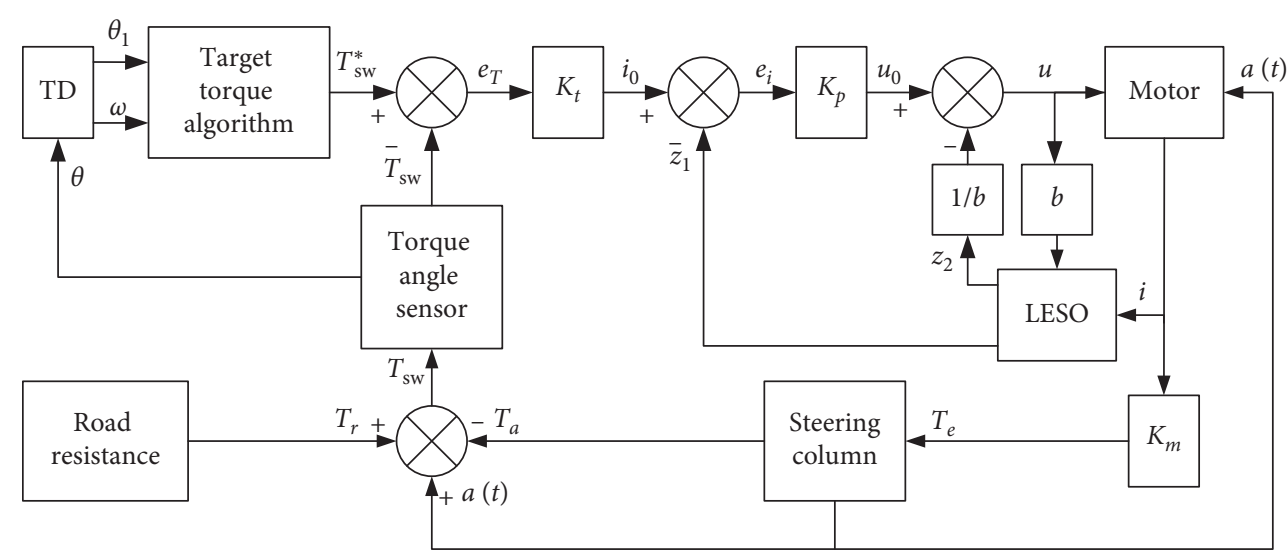

FIGURE 2: Block diagram of EPS motor control based on ADRC.

Therefore, a variable $\alpha$ can be defined as a first-order inertial link of $\theta$, which can be expressed as

$$
\alpha(s)=\frac{\theta(s)}{T s+1} .
$$

When $T$ is small, it can be seen from the nature of Laplace that $\alpha$ can approximate the input signal $\theta$ lagging $T$ in the time domain. The approximation equation can be expressed as

$$
\omega_{c}(t) \approx \frac{1}{T}(\theta(t)-\theta(t-T)) .
$$

However, due to the disturbance of sensor measurement noise and controller sampling noise in practical applications, the angle signal $\theta$ is actually composed of the real angle signal $\theta_{a}$ and the disturbance angle signal $\theta_{n}$. Therefore, when the initial state of the system is 0 , the convolution property of Laplace shows that $\alpha$ can be expressed as

$$
\begin{aligned}
\alpha(t) & =\int_{0}^{\infty} e^{(1 / T)(t-\tau)}\left(\theta_{a}(\tau)+\theta_{n}(\tau)\right) \mathrm{d} \tau \\
& =\int_{0}^{\infty} e^{(1 / T)(t-\tau)} \theta_{a}(\tau) \mathrm{d} \tau+\int_{0}^{\infty} e^{(1 / T)(t-\tau)} \theta_{n}(\tau) \mathrm{d} \tau .
\end{aligned}
$$

In the second term of equation (12), the noise $\theta_{n}(\tau)$ is high-frequency sampling noise, and the average value is 0 . Thus, $\alpha$ can be rewritten as

$$
\alpha(t) \approx \int_{0}^{\infty} e^{(1 / T)(t-\tau)} \theta_{a}(\tau) \mathrm{d} \tau \approx \theta_{a}(t-T) .
$$

The angular speed equation can be expressed as

$$
\begin{aligned}
\omega_{c}(t) & =\frac{1}{T}\left(\theta_{a}(t)-\theta_{a}(t-T)+\theta_{n}(t)\right) \\
& =\dot{\theta}_{a}(t)+\frac{1}{T} \theta_{n}(t) .
\end{aligned}
$$

As can be seen from equation (14), the smaller the time constant $T$, the larger the noise amplification. In practical applications, the system step size is usually less than $1 \mathrm{~ms}$, and the noise amplification is serious, which causes the real signal to be drowned.
In this paper, in order to reduce the noise amplification, TD is used to calculate the angular speed as

$$
\omega(t) \approx \frac{\theta\left(t-\tau_{1}\right)-\theta\left(t-\tau_{2}\right)}{\tau_{2}-\tau_{1}}, \quad 0<\tau_{1}<\tau_{2},
$$

where $\omega$ is the approximate angular speed of the steering wheel calculated by $\mathrm{TD}$ and $\tau_{1}$ and $\tau_{2}$ are two adjacent moments. The transfer function of equation (15) is

$$
\begin{aligned}
\omega(s) & =\frac{1}{\tau_{2}-\tau_{1}}\left(\frac{\theta(s)}{\tau_{1} s+1}-\frac{\theta(s)}{\tau_{2} s+1}\right) \\
& =\frac{s}{\tau_{1} \tau_{2} s^{2}+\left(\tau_{1}+\tau_{2}\right) s+1} \theta(s) .
\end{aligned}
$$

Both $\tau_{1}$ and $\tau_{2}$ can be approximately recorded as $\tau$ when the time point is very close. Then, equation (16) can be expressed as

$$
\omega(s)=\frac{r^{2} s}{s^{2}+2 r s+r^{2}} \theta(s)
$$

where $r$ is the reciprocal of $\tau$. After a simple equation change, equation (17) can be rewritten as

$$
\left\{\begin{array}{l}
\theta_{1}(s)=\frac{\omega(s)}{s} \\
\theta_{1}(s)=\frac{r^{2}}{s^{2}+2 r s+r^{2}} \theta(s),
\end{array}\right.
$$

where $\theta_{1}$ is the transition process of $\theta$. It can be seen from the nature of Laplace, $\theta_{1}$ is the output of $\theta$ through the second-order linear system, and $r$ is the damping parameter of the equation. When $r>1, \theta_{1}$ follows $\theta$ without overshoot. The state space realization of equation (18) can be expressed as

$$
\left\{\begin{array}{l}
\dot{\theta}_{1}(t)=\omega(t) \\
\dot{\omega}(t)=-r^{2}\left(\theta_{1}(t)-\theta(t)\right)-2 r \omega(t) \\
y=\omega(t)
\end{array}\right.
$$


Equation (19) is the general form of TD in the time domain, which has these characteristics:

(1) TD arranges $\theta_{1}$ as the transition process of $\theta$ to avoid the step impact caused by abrupt $\theta$ changes.

(2) The parameter $r$ affects the tracking speed of the transition process. The parameter $r$ is increasing with the demand of the control accuracy of the system becoming high.

(3) TD effectively reduces the influence of noise in the calculation of differential signal and improves the accuracy of differential signal.

Figure 3 shows the output of TD with different parameters in step response.

From Figure 3, when the parameter $r$ increases, $\theta_{1}$ can track $\theta$ faster and the approximate differentiation is more accurate. In this paper, $r=2500$.

To simulate the practical applications, add a white noise whose amplitude is $1 \%$ of the actual signal to the input signal. The system step size is set to $1 \mathrm{~ms}$. Figure 4 shows the output of the TD in the step response and the sinusoidal response.

From Figure 4, although the noise is only accounts for $1 \%$ of the signal, the differential error of conventional differentiators is too large to be applied. The differential error using TD is $0.3 \%$ of that of the conventional differential method. Therefore, a more accurate signal of steering wheel angular speed can be obtained by TD. Furthermore, a more accurate target torque algorithm can be obtained.

\subsection{Improved ADRC Strategy Design and Stability Analysis.} The basis of torque closed loop is to complete the current closed loop. The state equation of the motor is established as

$$
\left\{\begin{array}{l}
p i=-\frac{R_{0}}{L_{m}} i-\frac{R_{n}}{L_{m}} i-\frac{K_{e}}{L_{m}} \omega_{m}+\frac{1}{L_{m}} u, \\
y=i,
\end{array}\right.
$$

where $R_{0}$ is the static resistance of the motor; $R_{n}$ is the change in resistance when the motor is running; and $u$ and $i$ are the input and output of the equation of the state, respectively.

For the motor, the disturbance of the EPS system such as system friction and road resistance is finally reflected in the motor speed, which is difficult to accurately judge. Meanwhile, the motor parameters, such as motor resistance, change with the running state of the motor. Therefore, the input current of the motor is not only related to the motor voltage but also affected by internal and external disturbance. It needs three steps to realize the core idea of ADRC: (1) the internal and external disturbances of the system are regarded as the total disturbance; (2) the total disturbance is estimated by ESO; and (3) the estimated result is compensated by the closed-loop control link. So the state equation can be rewritten as

$$
\left\{\begin{array}{l}
p i=a_{0} i+f+b u, \\
y=i, \\
a_{0}=-\frac{R_{0}}{L_{m}}, \\
b=\frac{1}{L_{m}}, \\
f=-\frac{R_{n}}{L_{m}} i-\frac{K_{e}}{L_{m}} \omega_{m},
\end{array}\right.
$$

where $a_{0}$ and $b$ are the system parameters, respectively, and $f$ is the total disturbance. On the basis of equation (21), an ESO is established as

$$
\left\{\begin{array}{l}
e_{i}=z_{1}-i, \\
\dot{z}_{1}=z_{2}-\beta_{1} \cdot g\left(e_{i}\right)+a_{0} i+b u, \\
\dot{z}_{2}=-\beta_{2} \cdot g\left(e_{i}\right),
\end{array}\right.
$$

where $z_{1}$ is the estimate of the current $i ; z_{2}$ is the estimate of the total disturbance; $e_{i}$ is the error between the true value and the estimated value; $\beta_{1}$ and $\beta_{2}$ are the feedback gain coefficients, respectively; and $g\left(e_{i}\right)$ is a feedback function of the error. When $g\left(e_{i}\right)$ is a linear function, the LESO is established. When $g\left(e_{i}\right)$ is a nonlinear function, the NESO is established. The general nonlinear function is

$$
f a l\left(e_{i}, \alpha, \delta\right)= \begin{cases}\left|e_{i}\right|^{\alpha} \operatorname{sign}\left(e_{i}\right), & \left|e_{i}\right|>\delta, \\ \frac{e_{i}}{\delta^{1-\alpha}}, & \left|e_{i}\right| \leq \delta,\end{cases}
$$

where $\alpha$ and $\delta$ are the parameters of the function.

When the ESO estimation effect is good, the proportional control can achieve good current closed-loop control. Therefore, the total disturbance estimate is compensated proportionally, and the current closed-loop control equation is established as

$$
\left\{\begin{array}{l}
u_{0}=K_{P}\left(i_{0}-z_{1}\right), \\
u=u_{0}-\frac{z_{2}}{b},
\end{array}\right.
$$

where $i_{0}$ is the motor target current obtained from the target torque closed-loop control; $u_{0}$ is the proportional error feedback between $i_{0}$ and $z_{1} ; K_{p}$ is the proportionality coefficient; and $u$ is the real output voltage after disturbance compensation.

In fact, there is always an observation error between the estimate disturbance and the real disturbance:

$$
f^{\prime}=f-z_{1},
$$

where $f^{\prime}$ is the error of disturbance observation. When NESO is adopted and appropriate parameters are matched, $f^{\prime}$ is small and the system works well. However, the computation 


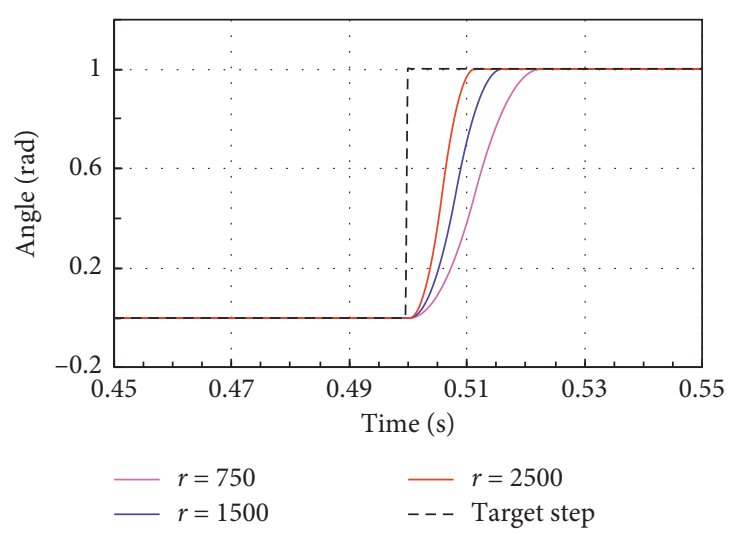

(a)

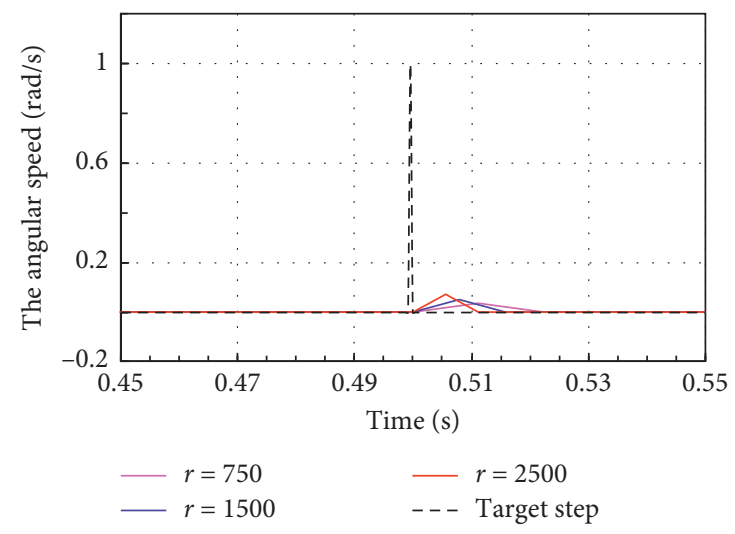

(b)

FIgURE 3: Output of the tracking differentiator. (a) The tracking process, $\theta_{1}$. (b) The approximately differential, $\omega$.

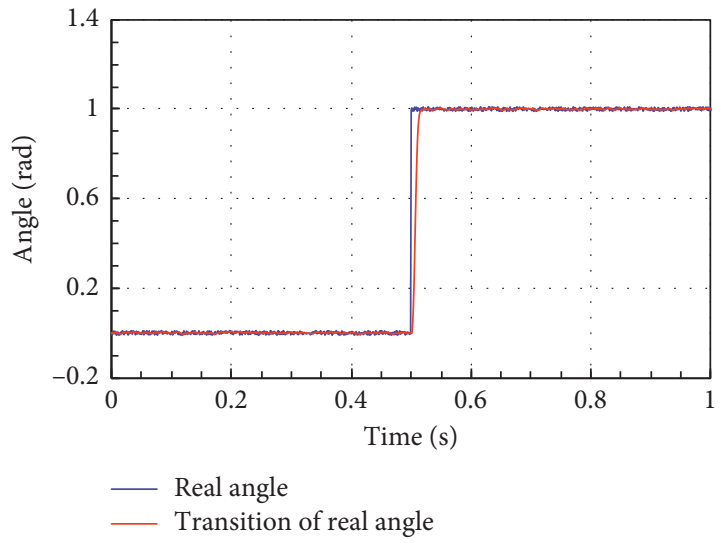

(a)

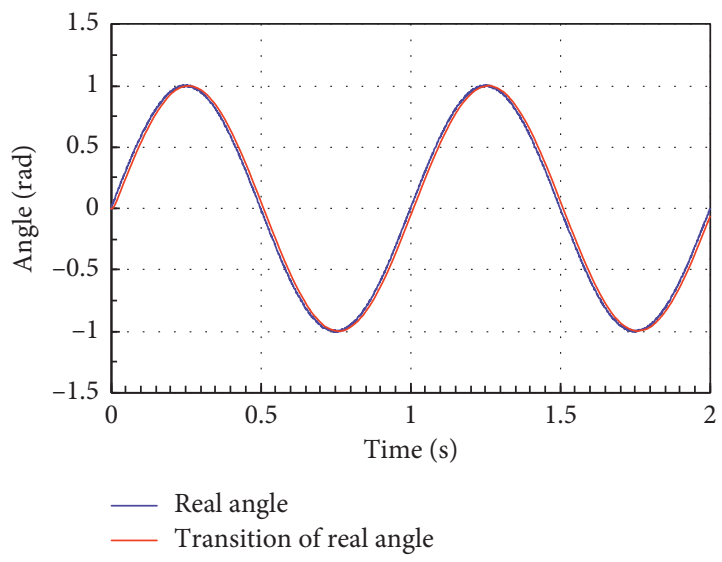

(c)

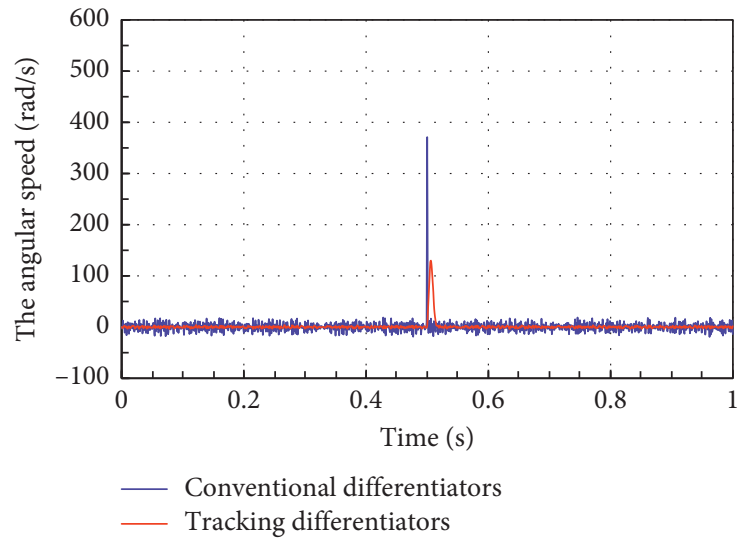

(b)

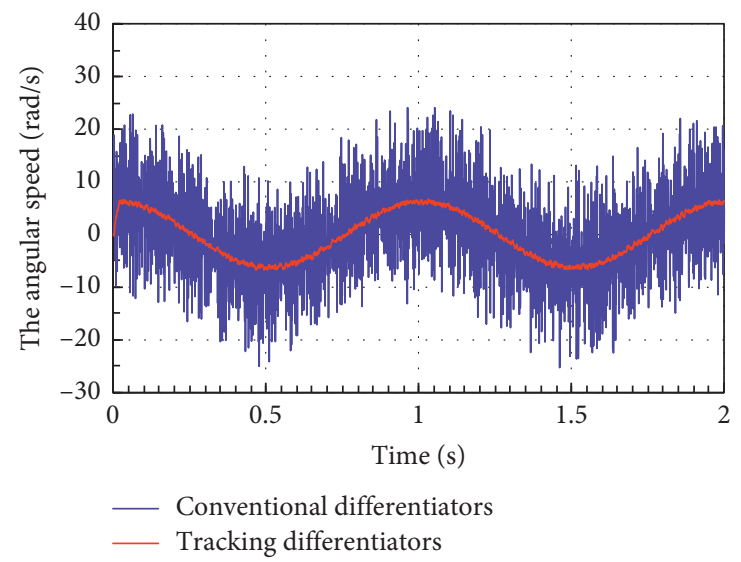

(d)

Figure 4: Output of TD. (a) Angle output under step response. (b) Angular speed output under step response. (c) Angle output under sinusoidal response. (d) Angular speed output under sinusoidal response.

of nonlinear function is very heavy in practical application. On the other hand, if LESO is adopted instead, the calculation is small, but $f^{\prime}$ is large when the target gain is large or the interference frequency is high. The responsiveness and anti-interference ability of ADRC is seriously affected.
Based on the principle of ADRC, the fundamental purpose of disturbance compensation is to transform the unknown system model into a first-order integration system which is easy to solve. Therefore, the error of disturbance observation is the difference between the target current and 
the output current. The target current is the first-order integral function of the target voltage:

$$
f^{\prime}(s)=\frac{b u_{0}(s)}{s+a_{0}}-i(s) .
$$

As long as $f^{\prime}$ is compensated, the estimation accuracy of disturbance can be improved. $f^{\prime}$ is considered the output of a system where the input $u_{2}$ is zero. Then, a new LESO2 for the system to estimate $f^{\prime}$ is established. The new LESO 2 and the original LESO1 form the parallel linear extended state observer (P-LESO). The total disturbance of the system is observed and compensated by LESO1, and the error of disturbance observation in LESO1 is observed and compensated by LESO2. Therefore, P-LESO observation method can reduce the error of disturbance observation and improve the dynamic responsiveness. The P-LESO is constructed as follows:

$$
\begin{aligned}
& \left\{\begin{array}{l}
e_{i}=z_{11}-i, \\
\dot{z}_{11}=z_{12}-\beta_{1} e_{i}+a_{0} i+b u, \\
\dot{z}_{12}=-\beta_{2} e_{i},
\end{array}\right. \\
& \left\{\begin{array}{l}
e_{i}^{\prime}=z_{21}-f^{\prime}, \\
\dot{z}_{21}=z_{22}-\beta_{1} e_{i}^{\prime}, \\
\dot{z}_{22}=-\beta_{2} e_{i}^{\prime},
\end{array}\right.
\end{aligned}
$$

where $z_{11}$ is the estimate of the output current; $z_{12}$ is the estimate of total system disturbance; $z_{21}$ is the estimate of $f^{\prime}$; and $\beta_{1}$, and $\beta_{2}$ are the tunable parameters, and their values will directly affect the fastness and accuracy of the disturbance estimation. Figure 5 shows the block diagram of EPS motor control based on the improved ADRC strategy using P-LESO.

The LESO1 is expressed in the form of state space:

$$
\left[\begin{array}{l}
\dot{z}_{1} \\
\dot{z}_{2}
\end{array}\right]=\left[\begin{array}{cc}
-\beta_{1} & 1 \\
-\beta_{2} & 0
\end{array}\right]\left[\begin{array}{l}
z_{11} \\
z_{12}
\end{array}\right]+\left[\begin{array}{c}
\beta_{1}-a_{0} \\
\beta_{2}
\end{array}\right] i+\left[\begin{array}{l}
b \\
0
\end{array}\right] u \text {. }
$$

The equation of the state is

$$
\Delta|s \mathbf{E}-\mathbf{A}|=s^{2}+\beta_{1} \cdot s+\beta_{2},
$$

where $\mathbf{A}=\left[\begin{array}{ll}-\beta_{1} & 1 \\ -\beta_{2} & 0\end{array}\right]$, which is the system matrix.

According to the Routh stability criterion, when $\beta_{1}>0$ and $\beta_{2}>0$, the observer must be stable. Meanwhile, the system matrix of LESO2 is the same form as LESO1, and $\mathrm{LESO} 2$ is also stable.

In EPS systems, the observer is required to estimate low frequency (within $10 \mathrm{~Hz}$ ) disturbance quickly and accurately. Therefore, the observer bandwidth should be above $100 \mathrm{~Hz} . \delta_{0}$ is the expected observer bandwidth. When $\beta_{1}=2 \delta_{0}$ and $\beta_{2}=\delta_{0}^{2}$, the expected observer characteristic equation is

$$
\left(s+\delta_{0}\right)^{2}=s^{2}+2 \delta_{0} \cdot s+\delta_{0}^{2} .
$$

The initial state of the system is assumed to 0 , the P-LESO equation can be obtained by eliminating the intermediate variables after Laplace transformation:

$$
\frac{z_{12}(s)+z_{21}(s)}{f(s)}=\frac{\delta_{0}^{2}}{\left(s+\delta_{0}\right)^{2}} .
$$

According to equation (32), the estimate disturbance and the real disturbance can be abstracted as a secondorder oscillation link. Therefore, the estimate disturbance is obtained after performing the first-order low-pass filtering twice on the real disturbance. The cut-off frequency of each level of low-pass filter is $\delta_{0}$. However, when $\beta_{1}=2 \delta_{0}$ and $\beta_{2}=\delta_{0}^{2}$, the damping ratio is 1 , and the equation has two equal negative real roots $-\delta_{0}$. In practical applications, the parameter is set to overdamping to prevent overshoot in step response. In this paper, $\beta_{1}=250$ and $\beta_{2}=12,000$.

Laplace transform is performed on equations (26), and (29) to obtain the transfer function between the input voltage and the output current of the motor:

$$
\frac{i(s)}{u(s)}=\frac{s}{k s+\beta_{2}}+\frac{k b^{2}-b \beta_{1}}{k b s+\beta_{2}} .
$$

According to equation (33), the relationship between output current and input voltage in the improved ADRC strategy is composed of a first-order inertia link and a differential link. On the premise of ensuring the stability of the system, the output of the system can be corrected earlier to improve the responsiveness.

3.3. Simulation Analysis of Improved ADRC. According to the working situation of the motor in the EPS system, the signal frequency of torque and angle is within $3 \mathrm{~Hz}$ and the typical frequency of mechanical disturbance is between $10 \mathrm{~Hz}$ and $30 \mathrm{~Hz}$, which is the low-frequency disturbance.

Figure 6 shows the disturbance estimation of three kinds of ESO at different frequencies. When the disturbance is $1 \mathrm{~Hz}$, all three observers agree well with the disturbance. When the disturbance is $10 \mathrm{~Hz}$, the disturbance estimation of LESO has a phase difference of $45 \mathrm{deg}$ compared with the real disturbance, but P-LESO and NESO still follow well. When the disturbance is $30 \mathrm{~Hz}$, the disturbance estimation of LESO has a phase difference of 90 deg compared with the real disturbance, while the phase difference in P-LESO and NESO is 18 deg. Therefore, the designed P-LESO can stably and accurately estimate the typical frequency of mechanical disturbance.

Figure 7 shows the step responses of different strategies to typical frequency signals when there is disturbance noise.

From Figure 7, the ADRC based on different ESO is better than PI control in noise suppression and response speed, especially at higher signal frequencies, no matter which steps input signal frequency or disturbance frequency. When the signal frequency is low, the responsiveness of the three ADRC strategies is similar. When the signal frequency increases, the responsiveness and anti-interference ability of P-LESO is better than that of LESO, which is basically the same of NESO. 


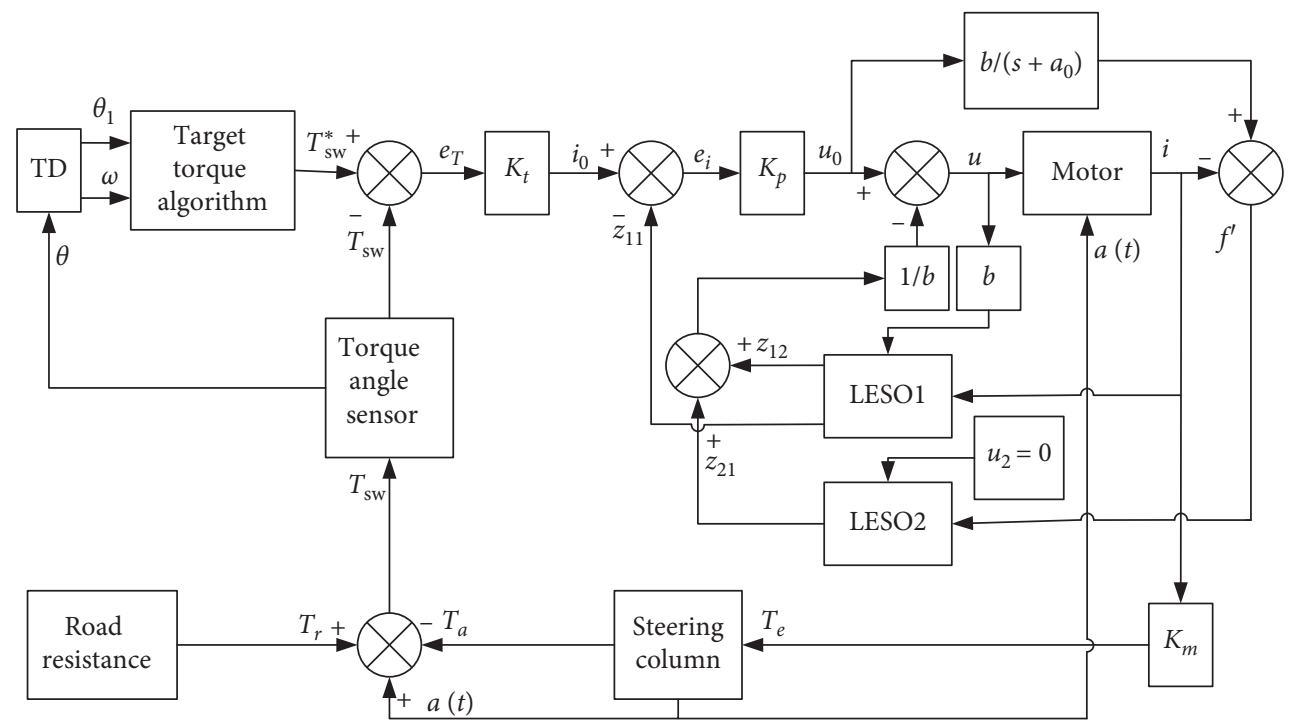

FIgURE 5: The block diagram of EPS motor control based on improved ADRC.

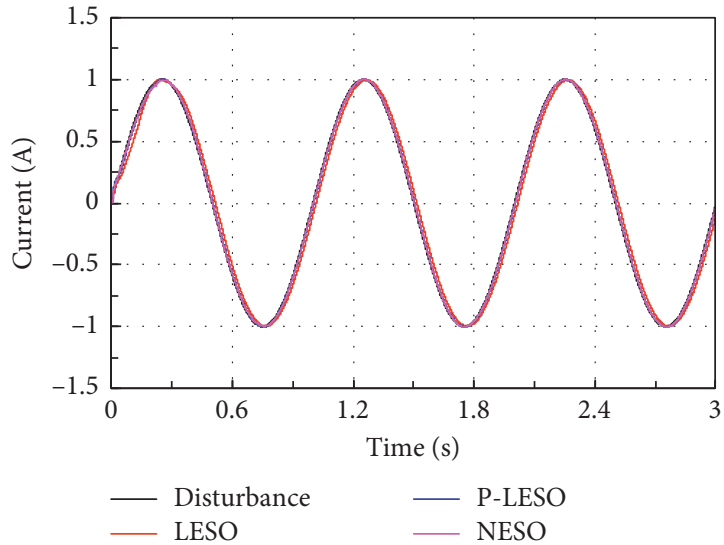

(a)

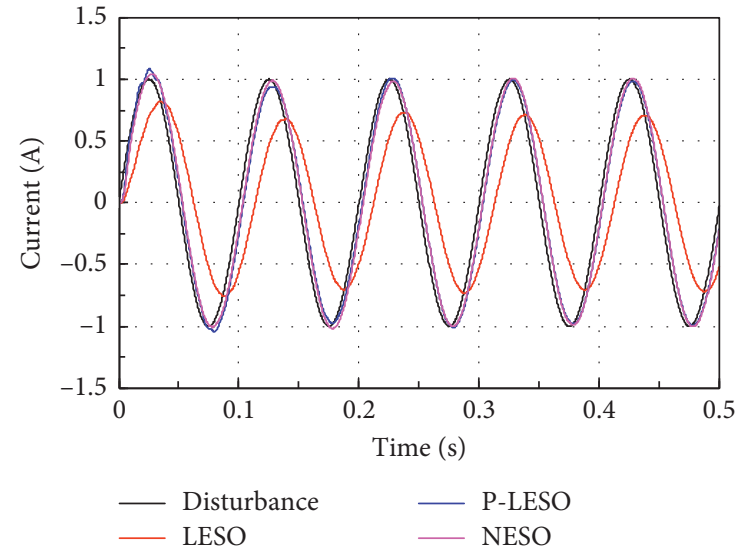

(b)

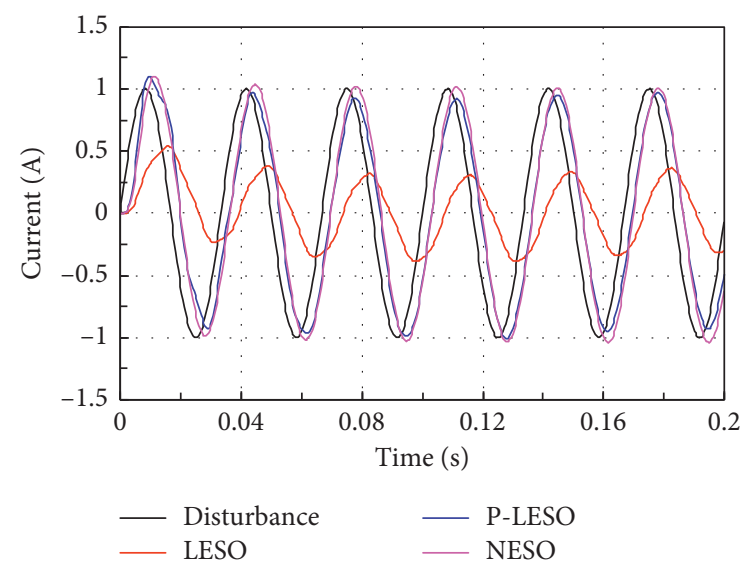

(c)

Figure 6: The disturbance estimation results of three kinds of ESO. (a) $1 \mathrm{~Hz}$ disturbance. (b) $10 \mathrm{~Hz}$ disturbance. (c) $30 \mathrm{~Hz}$ disturbance. 


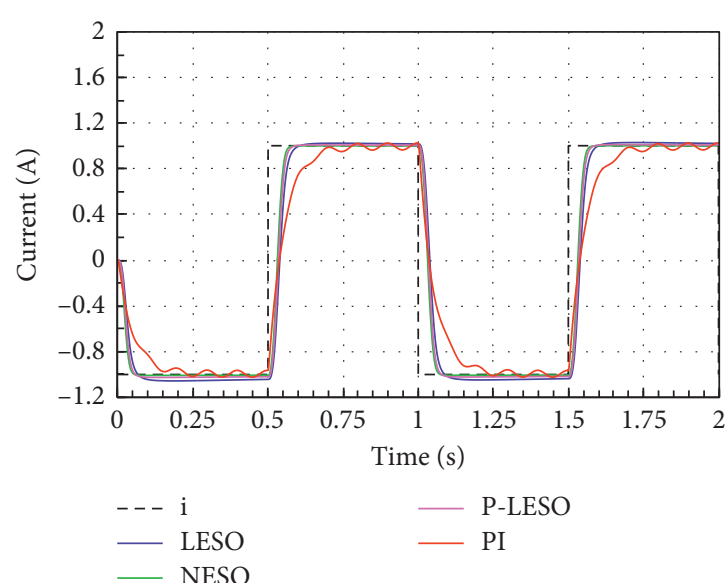

(a)

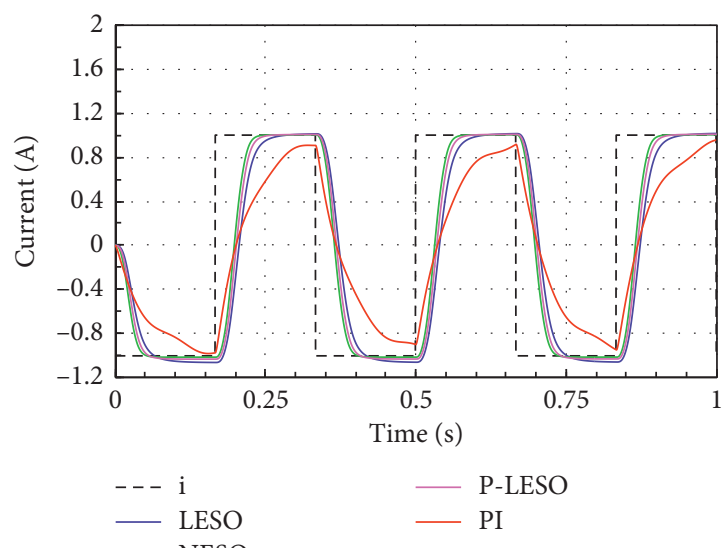

(c)

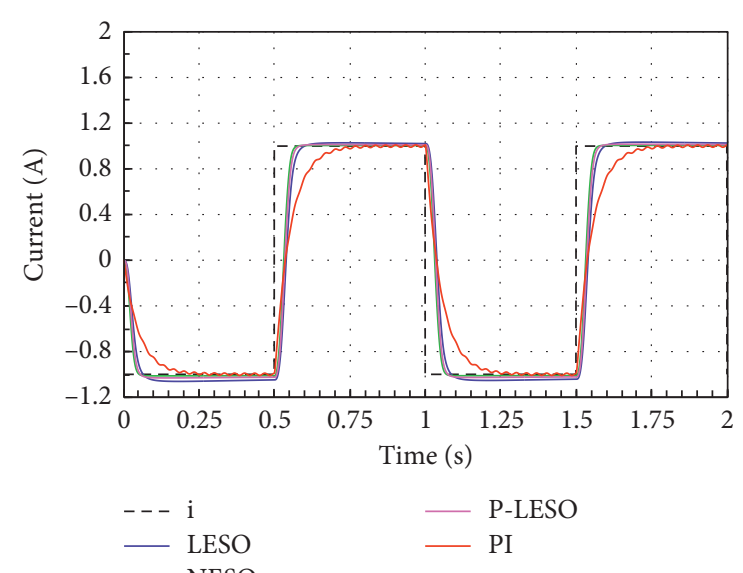

(b)

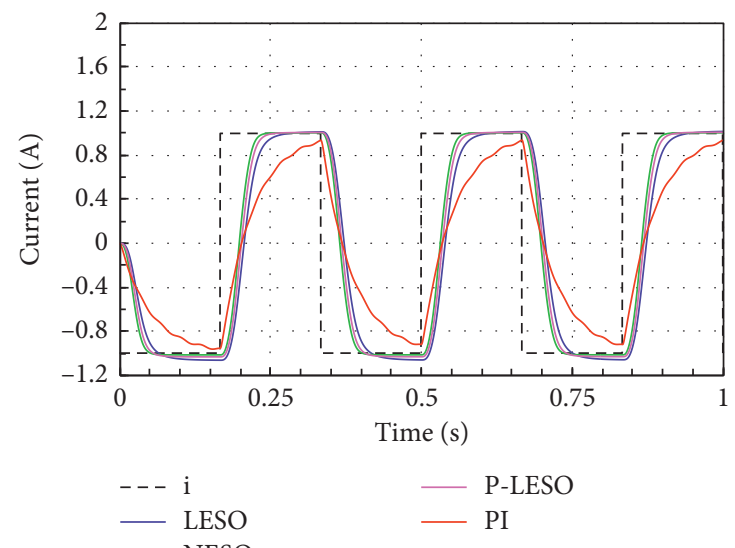

(d)

Figure 7: The result of the system step response. (a) $10 \mathrm{~Hz}$ noise and $1 \mathrm{~Hz}$ signal. (b) $30 \mathrm{~Hz}$ noise and $1 \mathrm{~Hz}$ signal. (c) $10 \mathrm{~Hz}$ noise and $3 \mathrm{~Hz}$ signal. (d) $30 \mathrm{~Hz}$ noise and $3 \mathrm{~Hz}$ signal.

\section{Control Strategy Experiment}

On the basis of theory and simulation analysis, the control performances of steering torque and motor current in improved ADRC are verified by vehicle experiment. In order to significantly compare the various control strategies, an experiment vehicle with large and irregular friction in the steering system is selected, and the experiment vehicle has multiple points of discontinuous resistance torque in the entire steering range. The disturbance is especially obvious on the uneven road, and the vehicle keeps running at a low speed. The rotation range of the steering wheel may be wide, but the steering wheel speed generally does not exceed $1 \mathrm{r} / \mathrm{s}$. Considering the deceleration ratio, the maximum speed of the motor does not exceed $1000 \mathrm{r} / \mathrm{min}$. The steering system parameters of the experiment vehicle are shown in Table 2.

The motor control strategy of the improved ADRC is realized by a cortex-M0 kernel motor controller. The control step size of the system is $1 \mathrm{~ms}$, and the driving frequency of PWM is $20 \mathrm{kHz}$. The experiment vehicle is shown in Figure 8 , including the steering system, embedded hardware controller, J-Link debugger, and host computer.
TABLE 2: Main parameters of the experiment vehicle.

\begin{tabular}{lcc}
\hline Parameters & Unit & Value \\
\hline Front axle load & $\mathrm{kg}$ & 635 \\
Reduction ratio & - & 16.5 \\
The motor rating & $\mathrm{W}$ & 270 \\
Maximum torque of motor & $\mathrm{N} \cdot \mathrm{m}$ & 2.4 \\
Steering wheel rotation range & $\mathrm{deg}$ & -720 to 720 \\
\hline
\end{tabular}

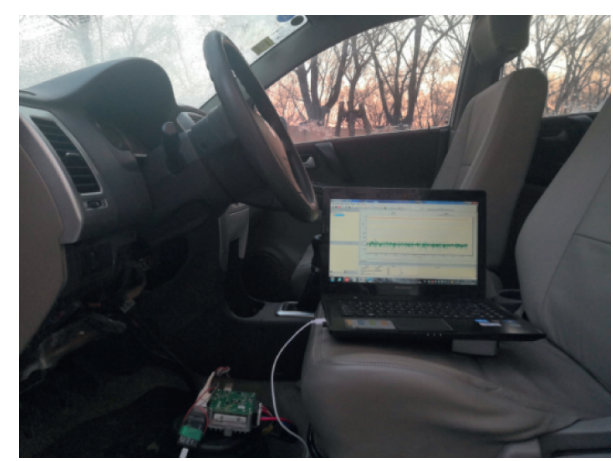

Figure 8: Experiment vehicle. 


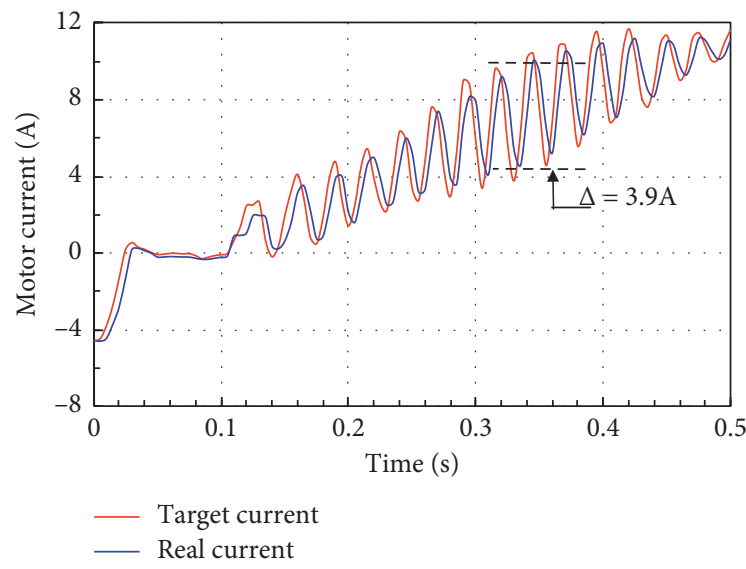

(a)

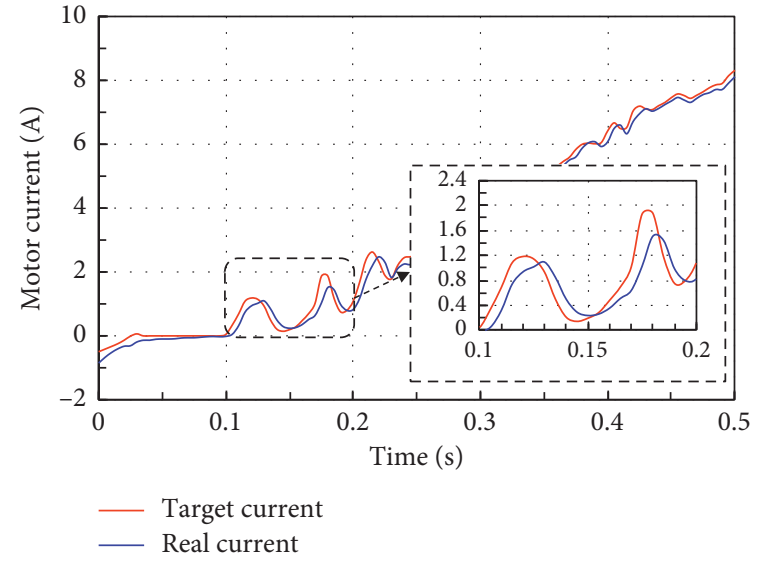

(b)

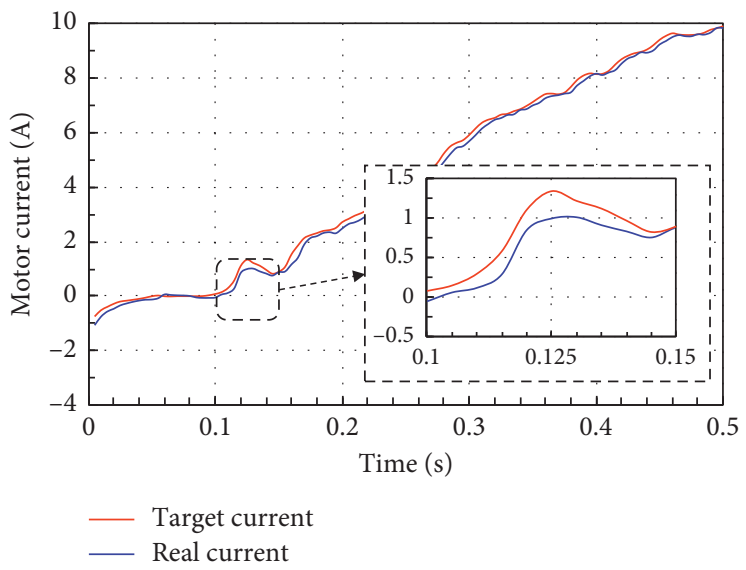

(c)

FIgure 9: Motor current response. (a) PI strategy. (b) ADRC strategy. (c) Improved ADRC strategy.

Experiment 1. The experiment vehicle is parked on the irregular rolling road, whose steering wheel is rotated from a resistance torque discontinuity point at a constant speed $1 \mathrm{r} /$ s. Then, the relationship between target current and real current in different control strategies is recorded in Figure 9.

In Figure 9(a), when the PI strategy is adopted with high target gain, the disturbance caused by irregular friction can be amplified in the closed-loop process, resulting in the current oscillation amplitude reaching about $4 \mathrm{~A}$. In Figure 9(b), the torque oscillation using the ADRC strategy can restore the stability within $0.13 \mathrm{~s}$, and the oscillation amplitude is reduced to $1.5 \mathrm{~A}$. In Figure 9(c), the torque oscillation using improved ADRC can restore stability within $0.05 \mathrm{~s}$, and the oscillation amplitude does not exceed $1 \mathrm{~A}$. Compared with the PI and ADRC strategy, the motor current using the improved ADRC strategy is more stable.

Experiment 2. The experiment vehicle is parked on the irregular rolling road. The steering wheel is rotated in the full range of travel at different rotation speed. Then, the curves of angle and torque of the steering wheel can be obtained and shown in Figure 10.
Figure 10(a) shows that when the steering wheel rotates at low speed, the steering torque fluctuation using the PI strategy increases to $2.5 \mathrm{~N} \cdot \mathrm{m}$. The steering torque fluctuation using the two ADRC strategies is less than $1 \mathrm{~N} \cdot \mathrm{m}$. The responsiveness of two ADRC strategies is better than the PI strategy, which makes the steering process smoother. In particular, when the steering wheel is rotated in the range of 400 to $500 \mathrm{deg}$, the steering torque using the PI strategy exceeds $4 \mathrm{~N} \cdot \mathrm{m}$, and the steering torque using the two ADRC strategies is $3 \mathrm{~N} \cdot \mathrm{m}$. The steering torque using the PI strategy is heavier than using the ADRC strategy, which affects the steering flexibility.

Figure 10(b) shows that when the steering wheel is rotated at a high speed, the torque fluctuation using the PI strategy and ADRC strategy increases to $3.5 \mathrm{~N} \cdot \mathrm{m}$. When using the improved ADRC strategy, the torque fluctuation is still less than $1 \mathrm{~N} \cdot \mathrm{m}$. In the stage of large angle where the load torque fluctuates greatly, the steering torque using the PI strategy exceeds $5 \mathrm{~N} \cdot \mathrm{m}$, and the steering torque using the ADRC strategy is $4 \mathrm{~N} \cdot \mathrm{m}$. Compared to the ADRC strategy, the torque fluctuation using the improved ADRC strategy is $3 \mathrm{~N} \cdot \mathrm{m}$, which is decreased by $25 \%$. This indicates that when the load torque changes quickly and 


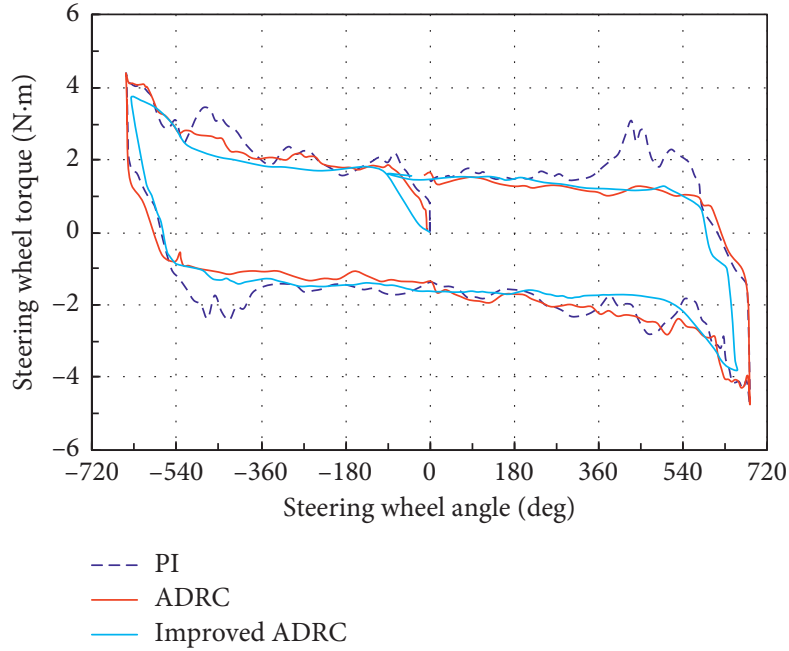

(a)

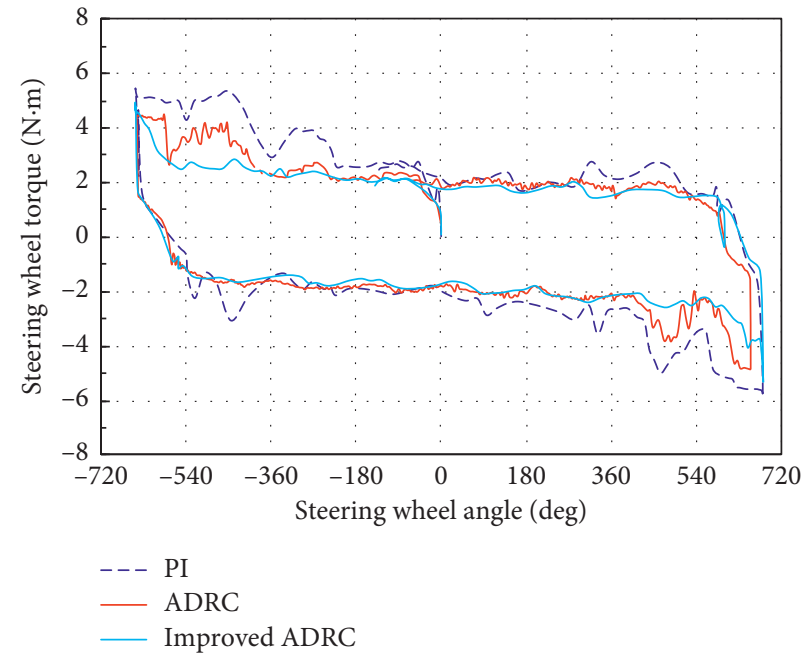

(b)

FIgURE 10: .Relationship between steering torque and steering angle. (a) The steering wheel speed is $0.2 \mathrm{r} / \mathrm{s}$. (b) The steering wheel speed is $1 \mathrm{r} / \mathrm{s}$.

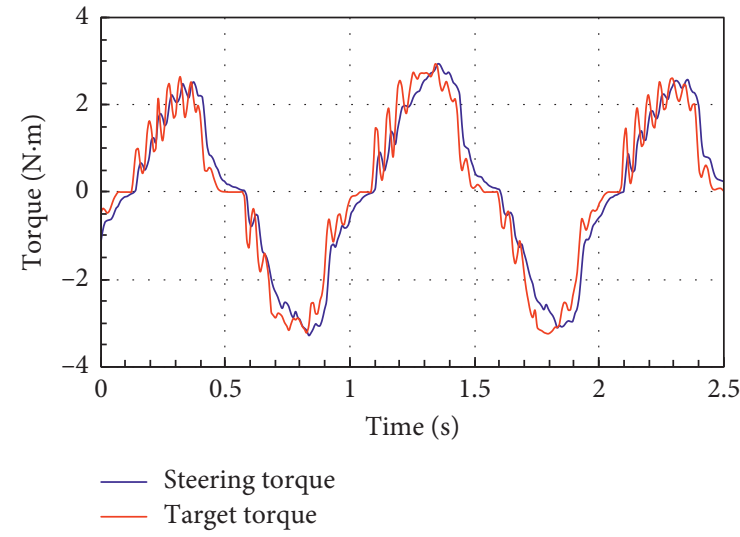

(a)

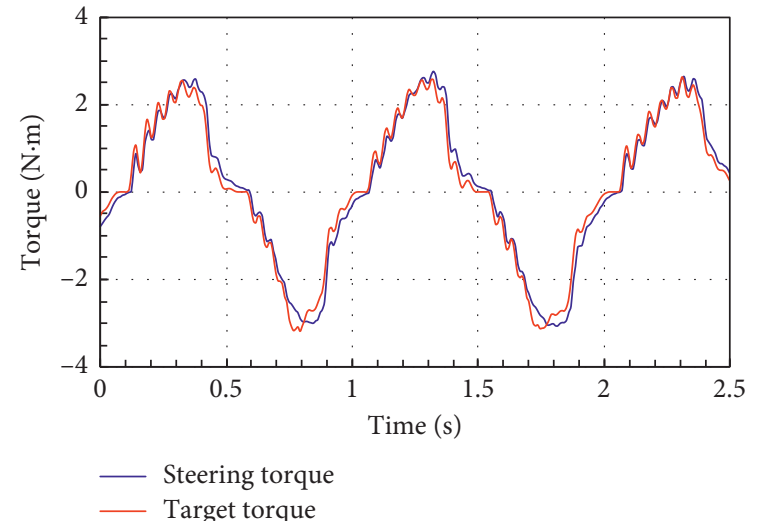

(b)

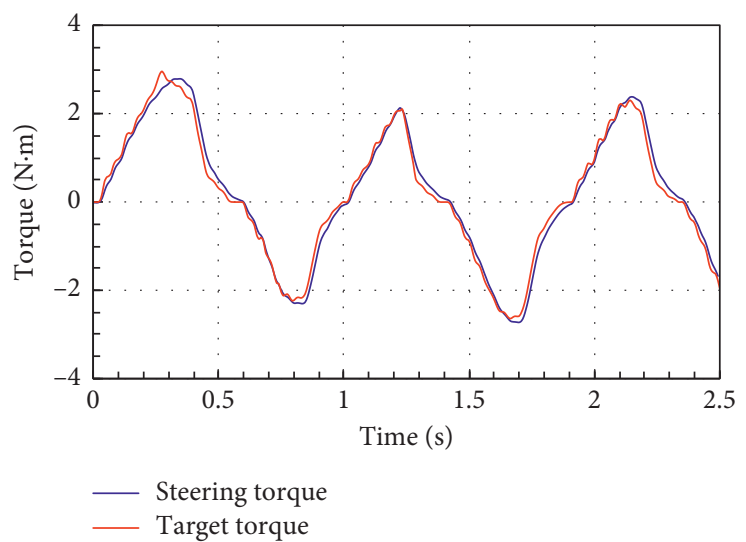

(c)

FIgURE 11: Variation of steering wheel torque. (a) PI strategy. (b) ADRC strategy. (c) Improved ADRC strategy.

severely, the PI and ADRC strategies cannot provide sufficient assisting torque in a timely manner. The improved ADRC is still responsive to the requirements of assist torque, which can keep the steering torque smooth and flexible.
Experiment 3. For the sake of safety, the vehicle speed is forced at $20 \mathrm{~km} / \mathrm{h}$. The steering wheel is rotated left and right repeatedly at the speed of $0.5 \mathrm{r} / \mathrm{s}$ to keep the vehicle's driving route approximately sinusoidal. The curve of steering wheel torque with time is shown in Figure 11. 
Figures 11(a) and 11(b) indicate the steering torque fluctuation violently due to irregular friction and unknown road resistance. The torque fluctuation using the PI strategy and ADRC strategy reaches above $1 \mathrm{~N} \cdot \mathrm{m}$ and $0.5 \mathrm{~N} \cdot \mathrm{m}$, respectively. Figure 11(c) shows that the torque fluctuation using improved ADRC is lower than $0.2 \mathrm{~N} \cdot \mathrm{m}$. Compared the three control strategies, the improved ADRC strategy shows good torque stability.

\section{Conclusion}

This paper proposes a new method for torque feedback control of the EPS motor, which has the advantages of strong anti-interference ability, smooth torque control, and small computation. TD is used to calculate the angular speed of the steering wheel to solve the problem of noise amplification in differential calculation. The improved ADRC strategy based on the P-LESO method is designed to avoid the use of nonlinear functions and reduce the computational burden of the system.

Simulation results show that the improved ADRC strategy can effectively reduce the low-frequency disturbance inside and outside the system. The control process has better responsiveness and anti-interference.

The vehicle experiment shows that the steering torque control method of the improved ADRC strategy reduces the oscillation of motor current and makes the steering wheel torque more flexible, smooth, and stable when compared with the PI strategy and ADRC strategy.

In the case of road resistance, mechanical friction, and motor parameter changes, the proposed method can still keep the steering torque of the vehicle in an appropriate and stable range.

\section{Data Availability}

The data used to support the findings of this study are included within the article.

\section{Conflicts of Interest}

The authors declare no conflicts of interest.

\section{Acknowledgments}

This work was supported by grants from the National Science Foundation of China (grant number 51575097) and the Fundamental Research Funds for the Central Universities (China) (no. 2572016AB72).

\section{References}

[1] M. Cheng, L. Sun, G. Buja, and L. Song, "Advanced electrical machines and machine-based systems for electric and hybrid vehicles,” Energies, vol. 8, no. 9, pp. 9541-9564, 2015.

[2] D. Lee, K.-S. Kim, and S. Kim, "Controller design of an electric power steering system," IEEE Transactions on Control Systems Technology, vol. 26, no. 2, pp. 748-755, 2018.

[3] W. Kim, Y. S. Son, and C. C. Chung, "Torque-overlay-based robust steering wheel angle control of electrical power steering for a lane-keeping system of automated vehicles,"
IEEE Transactions on Vehicular Technology, vol. 65, no. 6, pp. 4379-4392, 2016.

[4] Z. Z. Jiang and B. X. Xiao, "Electric power steering system control strategy based on robust $\mathrm{H}_{\infty}$ control for electric forklift," Mathematical Problems in Engineering, vol. 2018, Article ID 7614304, 2018.

[5] T. Yang, "A new control framework of electric power steering system based on admittance control," IEEE Transactions on Control Systems Technology, vol. 23, no. 2, pp. 762-769, 2015.

[6] A. Marouf, M. Djemai, C. Sentouh, and P. Pudlo, "A new control strategy of an electric-power-assisted steering system," IEEE Transactions on Vehicular Technology, vol. 61, no. 8, pp. 3574-3589, 2012.

[7] S. Fankem, T. Weiskircher, and S. Müller, "Model-based rack force estimation for electric power steering," IFAC Proceedings Volumes, vol. 47, no. 3, pp. 8469-8474, 2014.

[8] J.-H. Kim and J.-B. Song, "Control logic for an electric power steering system using assist motor," Mechatronics, vol. 12, no. 3, pp. 447-459, 2002.

[9] M. K. Hassan, N. A. M. Azubir, H. M. I. Nizam, S. F. Toha, and B. S. K. K. Ibrahim, "Optimal design of electric power assisted steering system (EPAS) using GA-PID method," Procedia Engineering, vol. 41, pp. 614-621, 2012.

[10] R. A. Hanifah, S. F. Toha, M. K. Hassan, and S. Ahmad, "Power reduction optimization with swarm based technique in electric power assist steering system," Energy, vol. 102, pp. 444-452, 2016.

[11] X. Chen, T. B. Yang, X. Q. Chen, and K. M. Zhou, “A generic model-based advanced control of electric power-assisted steering systems," IEEE Transactions on Control Systems Technology, vol. 16, no. 6, pp. 1289-1300, 2008.

[12] C. Dannöhl, S. Müller, and H. Ulbrich, " $\mathrm{H}_{\infty}$-control of a rackassisted electric power steering system," Vehicle System Dynamics, vol. 50, no. 4, pp. 527-544, 2012.

[13] D. Lee, K. Yi, S. Chang, B. Lee, and B. Jang, "Robust steeringassist torque control of electric-power-assisted-steering systems for target steering wheel torque tracking," Mechatronics, vol. 49, pp. 157-167, 2018.

[14] Y.-C. Hung, F.-J. Lin, J.-C. Hwang, J.-K. Chang, and K.-C. Ruan, "Wavelet fuzzy neural network with asymmetric membership function Controller for electric power steering System via improved differential evolution," IEEE Transactions on Power Electronics, vol. 30, no. 4, pp. 2350-2362, 2015.

[15] D. Saifia, M. Chadli, H. R. Karimi, and S. Labiod, "Fuzzy control for Electric Power Steering System with assist motor current input constraints," Journal of the Franklin Institute, vol. 352, no. 2, pp. 562-576, 2015.

[16] R. Chabaan and L. Y. Wang, "Control of electrical power assist systems: Hâ design, torque estimation and structural stability," JSAE Review, vol. 22, no. 4, pp. 435-444, 2001.

[17] C. Y. Wang, W. Z. Zhao, and L. Y. Yu, " $\mathrm{H}_{\infty}$ control for electric power steering road feel based on frequency shaping," Journal of Jilin University (Engineering and Technology Edition), vol. 41, no. 6, pp. 1532-1536, 2011.

[18] K. Moriwaki, "Autonomous steering control for electric vehicles using nonlinear state feedback control," Nonlinear Analysis: Theory, Methods \& Applications, vol. 63, no. 5-7, pp. 2257-2268, 2005.

[19] W. Zhao and C. Wang, "Mixed $\mathrm{H}_{2} / \mathrm{H}_{\infty}$ road feel control of EPS based on genetic algorithm," Science China Technological Sciences, vol. 55, no. 1, pp. 72-80, 2012.

[20] J. Han, "From PID to active disturbance rejection control," IEEE Transactions on Industrial Electronics, vol. 56, no. 3, pp. 900-906, 2009. 
[21] X. Ma, Y. Guo, and L. Chen, "Active disturbance rejection control for electric power steering system with assist motor variable mode," Journal of the Franklin Institute, vol. 355, no. 3, pp. 1139-1155, 2018

[22] M. B. Baharom, K. Hussain, and A. J. Day, "Design of full electric power steering with enhanced performance over that of hydraulic power-assisted steering," Proceedings of the Institution of Mechanical Engineers, Part D: Journal of Automobile Engineering, vol. 227, no. 3, pp. 390-399, 2013. 